\title{
NeuroImage
}

\section{Modelling event-related responses in the brain}

\author{
Olivier David, ${ }^{*}$ Lee Harrison, and Karl J. Friston \\ Wellcome Department of Imaging Neuroscience, Functional Imaging Laboratory, 12 Queen Square, London WC1N 3BG, UK
}

Received 22 April 2004; revised 30 September 2004; accepted 14 December 2004

Available online 16 February 2005

The aim of this work was to investigate the mechanisms that shape evoked electroencephalographic (EEG) and magneto-encephalographic (MEG) responses. We used a neuronally plausible model to characterise the dependency of response components on the models parameters. This generative model was a neural mass model of hierarchically arranged areas using three kinds of inter-area connections (forward, backward and lateral). We investigated how responses, at each level of a cortical hierarchy, depended on the strength of connections or coupling. Our strategy was to systematically add connections and examine the responses of each successive architecture. We did this in the context of deterministic responses and then with stochastic spontaneous activity. Our aim was to show, in a simple way, how event-related dynamics depend on extrinsic connectivity. To emphasise the importance of nonlinear interactions, we tried to disambiguate the components of event-related potentials (ERPs) or event-related fields (ERFs) that can be explained by a linear superposition of trial-specific responses and those engendered nonlinearly (e.g., by phase-resetting). Our key conclusions were; (i) when forward connections, mediating bottom-up or extrinsic inputs, are sufficiently strong, nonlinear mechanisms cause a saturation of excitatory interneuron responses. This endows the system with an inherent stability that precludes nondissipative population dynamics. (ii) The duration of evoked transients increases with the hierarchical depth or level of processing. (iii) When backward connections are added, evoked transients become more protracted, exhibiting damped oscillations. These are formally identical to late or endogenous components seen empirically. This suggests that late components are mediated by reentrant dynamics within cortical hierarchies. (iv) Bilateral connections produce similar effects to backward connections but can also mediate zero-lag phase-locking among areas. (v) Finally, with spontaneous activity, ERPs/ERFs can arise from two distinct mechanisms: For low levels of (stimulus related and ongoing) activity, the systems response conforms to a quasi-linear superposition of separable responses to the fixed and stochastic inputs. This is consistent with classical assumptions that motivate trial averaging to suppress spontaneous activity and disclose the ERP/ ERF. However, when activity is sufficiently high, there are nonlinear interactions between the fixed and stochastic inputs. This interaction is

* Corresponding author. Present address: INSERM U594 Neuroimagerie Fonctionnelle et Métabolique, CHU-Pavillon B-BP 217, 38043 Grenoble Cedex 09, France. Fax: +33 476765896.

E-mail address: Olivier.David@ujf-grenoble.fr (O. David).

Available online on ScienceDirect (www.sciencedirect.com). expressed as a phase-resetting and represents a qualitatively different explanation for the ERP/ERF.

(C) 2004 Elsevier Inc. All rights reserved.

Keywords: Electroencephalography; Magnetoencephalography; Neural networks; Nonlinear dynamics; Causal modelling

\section{Introduction}

Classical event-related potentials (ERPs) and event-related fields (ERFs) have been used for decades as putative electrophysiological correlates of perceptual and cognitive operations. However, the exact neurobiological mechanisms underlying their generation are largely unknown. Recently, there has been a special interest in the distinction between evoked and induced responses. Evoked responses are disclosed by conventional averaging procedures, whereas the latter usually call for single-trial analyses of induced oscillations. In this paper, we used neuronal simulations to examine the mechanisms that underpin ERPs/ERFs. In a companion paper, we will examine induced responses using time-frequency analyses and other transforms of single-trial data.

\section{Neural mass models}

The complexity of neural networks generating MEG/EEG signals (DeFelipe et al., 2002; Thomson and Deuchars, 1997) is considerable. This means that MEG/EEG observation models rely upon simplifying assumptions and empirical priors (David and Friston, 2003; Freeman, 1978; Lopes da Silva et al., 1974; Robinson et al., 2001; Stam et al., 1999; Valdes et al., 1999; Van Rotterdam et al., 1982; Wendling et al., 2000). The primary aim of this paper is to describe a candidate forward model and establish its face validity. This model was designed to reproduce responses seen empirically and enable mechanistic enquiries into the generation of evoked and induced responses. This is the focus of the current paper. However, we will also use this model in a forthcoming paper as an observation model, allowing its parameters to be inferred from real data (David et al., 2004b). In this context, face validity is especially important. 
Neural mass models of MEG/EEG usually comprise cortical macro-columns, which can be treated as surrogates for cortical areas and, sometimes, thalamic nuclei. These models use a small number of state variables to represent a neuronal population mean state. This approach, referred to loosely as a mean-field approximation, is efficient when determining the steady-state behaviour of neuronal systems but its accuracy in a dynamic or nonstationary context is less established (Haskell et al., 2001). However, we will assume that the mean field approximation is sufficient for our purposes. The majority of neural mass models of MEG/EEG have been designed to generate alpha rhythms (Jansen and Rit, 1995; Lopes da Silva et al., 1974; Stam et al., 1999; Van Rotterdam et al., 1982). Recent studies have shown that it is possible to reproduce the whole spectrum of MEG/EEG oscillations, using appropriate values of model parameters (David and Friston, 2003; Robinson et al., 2001). In addition, these models have been used to test specific hypotheses about brain function, e.g., focal attention (Suffczynski et al., 2001). Pathological activity such as epilepsy can also be emulated. This means, in principle, that generative models of the sort employed above could be used to characterise the pathophysiological mechanisms underlying seizure activity (Robinson et al., 2002; Wendling et al., 2002).

To date, modelling event-related activity using neural mass models has received much less attention. An early attempt, in the context of visual ERPs, showed that it was possible to emulate ERP-like damped oscillations (Jansen and Rit, 1995). A more sophisticated thalamo-cortical model has been used to simulate event-related synchronisation (ERS) and event-related desynchronisation (ERD), commonly found in the alpha band (Suffczynski et al., 2001). Finally, it has been shown that model parameters can be adjusted to fit real ERPs (Rennie et al., 2002). These studies (Rennie et al., 2002; Suffczynski et al., 2001) emphasise the role of the thalamo-cortical interactions by modelling the cortex as a single compartment.

\section{Hierarchical models}

It is well-known that the cortex has a hierarchical organisation (Crick and Koch, 1998; Felleman and Van Essen, 1991), comprising bottom-up, top-down and lateral processes that can be understood from an anatomical and cognitive perspective (Engel et al., 2001). We have previously discussed the importance of hierarchical processes, in relation to perceptual inference in the brain, using the intimate relationship between hierarchical models and empirical Bayes (Friston, 2002). The current work was more physiologically motivated. Using a hierarchical neural mass model, we were primarily interested in the effects, on event-related MEG/ EEG activity, of connections strengths, and how these effects were expressed at different hierarchical levels. In addition, we were interested in how nonlinearities in these connections might be expressed in observed responses.

The neuronal model described below embodies many neuroanatomic and physiological constraints which lend it a neuronal plausibility. It has been designed to (i) explore emergent behaviours that may help understand empirical phenomena and, critically, (ii) as the basis of dynamic observation models. Although the model comprises coupled systems, the coupling is highly asymmetric and heterogeneous. This contrasts with homogenous and symmetrically coupled map lattices (CML) and globally coupled maps (GCM) encountered in more analytic treatments. Using the concepts of chaotic dynamical systems, GCMs have motivated a view of neuronal dynamics that is cast in terms of high-dimensional transitory dynamics among 'exotic' attractors (Tsuda, 2001). Much of this work rests on uniform coupling, which induces a synchronisation manifold, around which the dynamics play. The ensuing chaotic itinerancy has many intriguing aspects that can be related to neuronal systems (Breakspear et al., 2003; Kaneko and Tsuda, 2003). However, the focus of this work is not chaotic itinerancy but chaotic transience (the transient dynamics evoked by perturbations to the systems state) in systems with asymmetric coupling. This focus precludes much of the analytic treatment available for GCMs (but see Jirsa and Kelso, 2000 for an analytical description of coherent pattern formation in a spatially continuous neural system with a heterogeneous connection topology). However, as we hope to show, simply integrating the model, to simulate responses, can be a revealing exercise.

\section{Mechanisms of ERP/ERF generation; linear or nonlinear?}

It is generally held that an ERP/ERF is the result of averaging a set of discrete stimulus-evoked brain transients (Coles and Rugg, 1995). However, several groups (Jansen et al., 2003; Klimesch et al., 2004; Kolev and Yordanova, 1997; Makeig et al., 2002) have suggested that some ERP/ERF components might be generated by stimulus-induced changes in ongoing brain dynamics. This is consistent with views emerging from several neuroscientific fields, suggesting that phase-synchronisation, of ongoing rhythms, across different spatio-temporal scales mediates the functional integration necessary to perform higher cognitive tasks (Penny et al., 2002; Varela et al., 2001). In brief, a key issue is the distinction between processes that do and do not rely on phase-resetting of ongoing spontaneous activity. Both can lead to the expression of ERP/ERF components but their mechanisms are very different.

EEG and MEG signals are effectively ergodic and cancel when averaged over a sufficient number of randomly chosen epochs. The fact that ERPs/ERFs exhibit systematic waveforms, when the epochs are stimulus locked, suggests either a reproducible stimulus-dependent modulation of amplitude or phase-locking of ongoing MEG/EEG activity (Tass, 2003). The key distinction, between these two explanations, is whether the stimulus-related component interacts with ongoing or spontaneous activity. If there is no interaction, the spontaneous component will be averaged out, because it has no consistent phase relationship with stimulus onset. Conversely, if there is an interaction, dominant frequencies of the spontaneous activity must experience a phase-change, so that they acquire a degree of phase-locking to the stimulus. Note that phaseresetting is a stronger-requirement than induced oscillations. It requires any induced dynamics to be phase-locked in peristimulus time. In short, phase-resetting is explicitly nonlinear and implies an interaction between stimulus-related response and ongoing activity. Put simply, this means that the event-related response depends on ongoing activity. This dependency can be assessed with the difference between responses elicited with and without the stimulus (if we could reproduce exactly the same ongoing activity). In the absence of interactions, there will be no difference. Any difference implies nonlinear interactions. Clearly, this cannot be done empirically but it can be pursued using neuronal simulations.

The secondary aim of the current work was to use realistic neural mass models of hierarchically organised cortical areas to see whether phase-resetting is an emergent phenomenon and a plausible candidate for causing ERPs/ERFs. Phase-resetting is used in this paper as an interesting example of nonlinear responses 
that have been observed empirically. We use it to show that nonlinear mechanisms can be usefully explored with neuronal models of the sort developed here. In particular, static nonlinearities, in neuronal mass models, are sufficient to explain phase-resetting. Phase-resetting represents nonlinear behaviour because, in the absence of amplitude changes, phase-changes can only be mediated in a nonlinear way. This is why phasesynchronisation plays a central role in detecting nonlinear coupling among sources (Breakspear, 2002; Tass, 2003).

\section{Overview}

This paper is structured as follows. In the first section, we introduce the hierarchical neural mass model used in the remaining sections. It is based on previous neuroanatomic studies by Felleman and van Essen (Felleman and Van Essen, 1991) and work by Jansen and Rit on modelling MEG/EEG data (Jansen and Rit, 1995). In the second section, we demonstrate the basic behaviour of the model, by successive elaboration of a cortical hierarchy. We start with forward connections and then add backward and lateral connections. The goal of this approach was to provide an intuitive understanding of MEG/EEG like dynamics generated by coupled nonlinear systems. These simulations were performed in the absence of spontaneous activity. In the third section, we examine the interaction between evoked and spontaneous activity, using a representative hierarchical architecture established in the previous section. Finally, we discuss the potential benefits of this modelling approach, for the study of measured MEG/EEG activity.

\section{Hierarchical models of event-related MEG/EEG activity}

\section{Cortico-cortical connections}

Although neural mass models originated in the early 1970s (Freeman, 1978; Lopes da Silva et al., 1974; Wilson and Cowan, 1972), none have addressed explicitly the hierarchical nature of cortical organisation. The minimal model we propose, which accounts for directed extrinsic connections, uses the rules in Felleman and Van Essen (1991). Extrinsic connections are connections that traverse white matter and connect cortical regions (and subcortical structures). These rules, based upon a tri- partitioning of the cortical sheet (into supra-, infra-granular layers and granular layer 4), have been derived from experimental studies of monkey visual cortex. We will assume that they can be generalised to the whole cortex. The ensuing model is general, and can be used to model various cognitive paradigms (David et al., 2004b). However, variability among different cytoarchitectonic regions is restricted to differences in physiological parameters, under the same microcircuitry. Under this simplifying assumption, the connections can be defined as in Fig. 1: (i) Bottom-up or forward connections originate in agranular layers and terminate in layer 4. (ii) Top-down or backward connections only connect agranular layers. (iii) Lateral connections originate in agranular layers and target all layers. All these long-range or extrinsic cortico-cortical connections are excitatory and are mediated through the axons of pyramidal cells.

Although the thalamo-cortical connections have been the focus of several modelling studies, they represent a minority of extrinsic connections: in contrast, it is thought that at least $99 \%$ of axons in white matter link cortical areas of the same hemisphere (Abeles, 1991). For this reason, and for simplicity, we do not include the thalamic nuclei in our model. However, they can be included for any application where the role of the thalamus (or other subcortical structure) is thought important.

\section{Jansen model of a cortical area}

The neocortex is commonly described as a 6-layered structure (DeFelipe et al., 2002). Spiny neurons (pyramidal cells and spiny stellate cells) and smooth neurons are the two major groups of cortical neurons. The majority of cortical neurons are pyramidal cells that are found in layers 2 to 6 . Most spiny stellate cells are interneurons that are located in the middle cortical layers. Smooth neurons are essentially GABAergic interneurons distributed in all layers. In general, cortical neurons are thought to be organised into multiple, small repeating microcircuits. In spite of cortical heterogeneity, a common basic microcircuit has emerged. Its skeleton is formed by a pyramidal cell which receives excitatory inputs that originate from extrinsic afferent systems and spiny cells. Inhibitory inputs originate mostly from GABAergic interneurons. These micro-anatomical characteristics have been found in all cortical areas and species examined so far and, therefore, they can be considered as fundamental aspects of cortical organisation (DeFelipe et al., 2002).
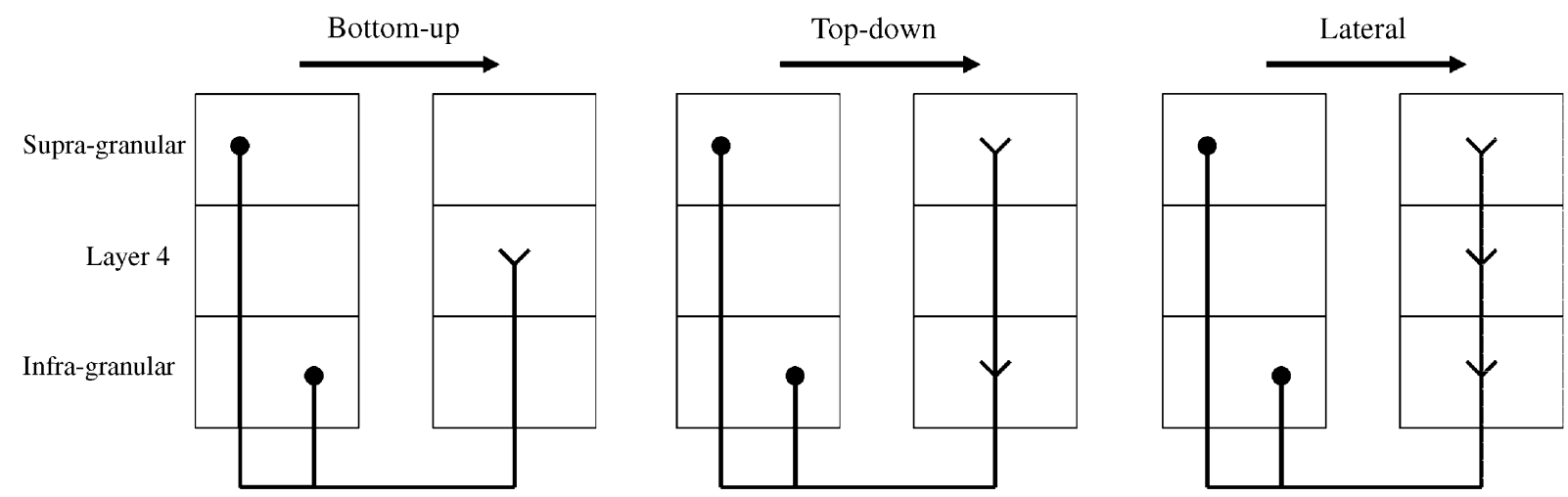

Fig. 1. Connection rules adopted for the construction of hierarchical models for MEG/EEG signals. These rules are a simplified version of those proposed by Felleman and Van Essen (1991). The cortical sheet is divided into two components: the granular layer (layer 4) and the agranular layers (supra- and infragranular layers). Bottom-up connections originate in agranular layers and terminate in layer 4. Top-down connections only engage agranular layers. Lateral connections originate in agranular layers and target all layers. 
The Jansen model (Jansen and Rit, 1995) follows the microcircuitry described above to emulate the MEG/EEG activity of a cortical area. Consequently, it is particularly well suited to embed in a hierarchical structure. A cortical area is modelled by three subpopulations. A population of excitatory pyramidal (output) cells receives inputs from inhibitory and excitatory populations of interneurons, via intrinsic connections (intrinsic connections are confined to the cortical sheet). Within this model, excitatory interneurons can be regarded as spiny stellate cells found predominantly in layer 4 and in receipt of forward connections (Miller, 2003). Excitatory pyramidal cells and inhibitory interneurons will be considered to occupy agranular layers and receive backward and lateral inputs.

We have described dynamics of these three subpopulations previously. We will review the model briefly but refer interested reader to David and Friston (2003) for more details. The main difference (cf. David and Friston, 2003) is that ERPs/ERFs are modelled as small perturbations around the resting potential. Therefore, all the variables below are zero-mean, centred on the resting state we assumed to be 0 . The evolution of population dynamics rests on two operators. The first transforms $p$, the average density of pre-synaptic input arriving at the population, into $v$, the average post-synaptic membrane potential. This is modelled by the linear transformation $v=h \otimes p$, where $\otimes$ denotes convolution and $h$ is the impulse response or kernel

$h(t)=\left\{\begin{array}{ll}\frac{H t \exp (-t / \tau)}{\tau} & t \geq 0 \\ 0 & t<0\end{array}\right.$.

The excitatory ( $e$ ) and inhibitory $(i)$ kernels, $h_{e}$ and $h_{i}$ respectively, are parameterised by $H_{e, i}$ and $\tau_{e, i}$ modelling specific properties of inhibition and excitation. The parameters $H_{e, i}$ control the maximum post-synaptic potential and $\tau_{e, i}$ are lumped time constants of passive membrane currents and other spatially distributed delays in the dendritic tree. The second operator transforms the average membrane potential of each subpopulation into an average firing rate. This is assumed to be instantaneous and is described by the sigmoid function

$S(v)=\frac{2 e_{0}}{1+\exp (-r v)}-e_{0}$

where $e_{0}$ and $r$ are parameters that determine its shape (e.g., voltage sensitivity). It is this function that endows the simulation with nonlinear behaviours that are critical for phenomena like phase-resetting.

Interactions, among the different subpopulations, depend on the constants $\gamma_{i}$, which control the strength of intrinsic connections and the total number of synapses expressed by each subpopulation. The relative values of these constants are fixed, using anatomical information from the literature, as described in Jansen and Rit (1995): $\gamma_{2}=0.8 \gamma_{1}, \gamma_{3}=\gamma_{4}=0.25 \gamma_{1}$. The Jansen model is summarised in Fig. 2.

We assume that MEG/EEG signals are a linear mixture of the average depolarisation of pyramidal cells. This mixture depends upon source lead-fields that model the spatial distribution of the ensuing electromagnetic fields (Baillet et al., 2001). We further assume that the depolarisation of pyramidal cells is proportional to the cortical current source densities, which are estimated using inverse solutions (Baillet et al., 2001). Therefore, we consider the indirectly observed 'output' of the Jansen model to be the depolarisation of pyramidal cells. For simplicity, we ignore the effects of instrumental amplifiers and assume that MEG/EEG cortical current densities can be estimated precisely and refer to them as "MEG/EEG signals." Thus, unless otherwise specified, signals are simply the depolarisation of pyramidal cells.

For given synaptic kernels $h$ and sigmoid functions $S$, the Jansen model can reproduce a large variety of MEG/EEG-like

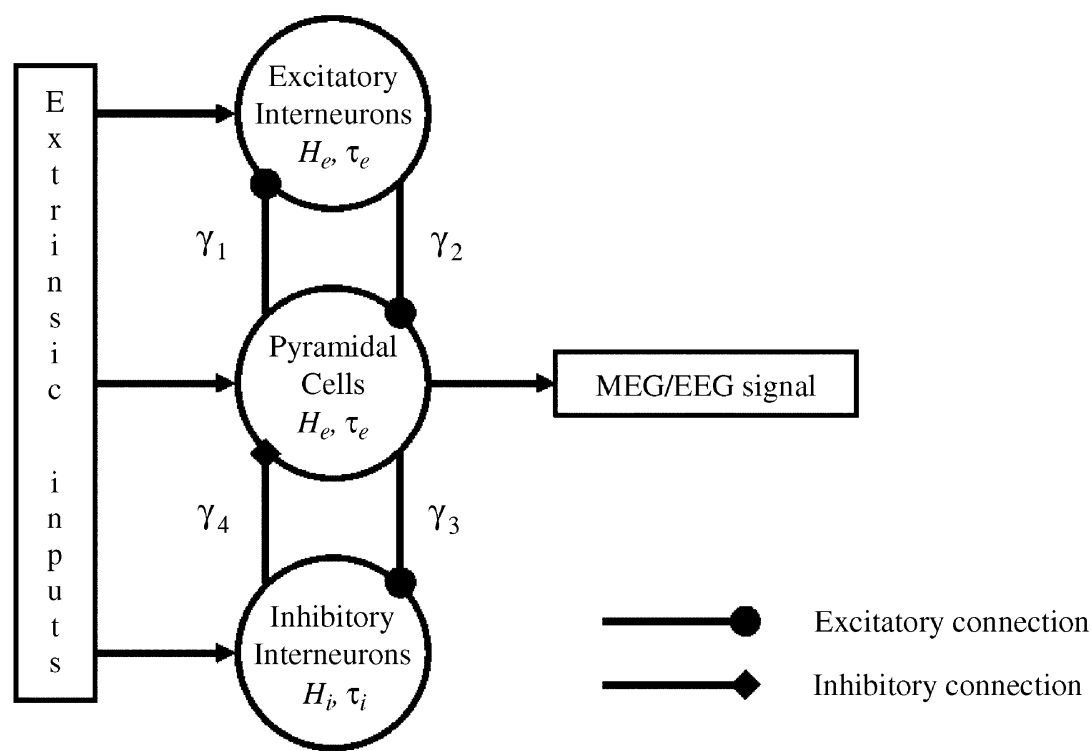

Fig. 2. Jansen's model of a cortical area. Three neuronal subpopulations are considered to model a cortical area. Pyramidal cells interact with both excitatory and inhibitory interneurons with the connectivity constants $\gamma_{2}=0.8 \gamma_{1}, \gamma_{3}=\gamma_{4}=0.25 \gamma_{1}$. The parameters $H_{e, i}$ and $\tau_{e, i}$ control the expression of post-synaptic potentials as shown in Eq. (1). We assume that the average depolarisation of pyramidal cells $y$ is proportional to reconstructed cortical current densities obtained with source reconstruction algorithms using MEG/EEG scalp data. For simplicity, we use the same term "MEG/EEG signal" for estimated cortical and measured scalp MEG/EEG data. 
waveforms (David and Friston, 2003; Jansen and Rit, 1995; Wendling et al., 2000). Although we have presented the dynamics in terms of a convolution operator and static nonlinearly, the integration of the model actually proceeds using the equivalent differential equations. These are provided in Appendix A. Being able to formulate the model in terms of differential equations is important for extending the neural mass formulation used here to a true mean field approximation using the Fokker-Plank formalism (De Groff et al., 1993; Fourcaud and Brunel, 2002). This will be the subject of a future communication (Harrison et al., submitted for publication).

\section{Hierarchical extension of the Jansen model}

Using the connection rules above, it is straightforward to construct hierarchical cortico-cortical networks using Jansen models of cortical areas. The different types of connections are shown in Fig. 3, in terms of connections among the three subpopulations. To model event-related responses, the network receives inputs via input connections. These connections are exactly the same as forward connections delivering fixed or stochastic inputs $u$ to the spiny stellate cells in layer 4 . In the present context, they can be regarded as connections from thalamic or geniculate nuclei. Inputs $u$ can model incoming stimuli and stochastic background activity. The influence of the $i^{\text {th }}$ input is controlled by the parameter $c_{i}$.

Connections among areas are mediated by long-range excitatory (glutaminergic) pathways. As discussed in Section 2.1, we consider three types of extrinsic connections (Fig. 3): forward, backward and lateral. The strength of each type of connection is controlled by a coupling parameter $a: a^{\mathrm{F}}$ for forward, $a^{\mathrm{B}}$ for backward and $a^{\mathrm{L}}$ for lateral. We model propagation delays for these connections (see Appendix A).

Using these connections, hierarchical cortical models for MEG/EEG can be constructed to test various hypotheses, and represent examples of dynamic causal models (Friston et al., 2003). The causal model here is a multiple-input multiple-output system that comprises $m$ inputs and $l$ outputs with one output per region. The $m$ inputs correspond to designed causes (e.g., stimulus functions encoding the occurrence of events) or stochastic processes modelling background activity. In principle, each input could have direct access to every region. However, in practice the effects of inputs are usually restricted to a single input region, usually the lowest in the hierarchy. Each of the $l$ regions produces a measured output that corresponds to the MEG/EEG signal. Each region has five $\left(H_{e, i}, \tau_{e, i}, \gamma_{1}\right)$ intrinsic parameters such as the membrane time constants described above. These play a crucial role in generating regional responses. However, in the present study, we will consider them fixed and focus on the extrinsic coupling parameters or effective connectivity. These are the matrices $\mathbf{C}, \mathbf{A}^{\mathrm{F}}, \mathbf{A}^{\mathrm{B}}$ and $\mathbf{A}^{\mathrm{L}}$ that contain the coupling parameters $c, a^{\mathrm{F}}, a^{\mathrm{B}}$ and $a^{\mathrm{L}}$. The values of these parameters, used in the following simulations, are provided in Appendix A and in figure captions.

\section{Input-output behaviour}

In this section, we characterise the input-output behaviour of a series of canonical networks in terms of their impulse response functions. This is effectively the response (mean depolarisation of pyramidal subpopulations) to a delta-function-input or impulse. The simulations of this section can be regarded modelling eventrelated responses to events of short duration, in the absence of spontaneous activity or stochastic input. In the next section, we will use more realistic inputs that comprise both stimulus-related and stochastic components.

\section{The effects of inputs}

Inputs $u$ act directly on the spiny stellate neurons of layer 4 . Their influence is mediated by the forward connections parameterised by the matrix $\mathbf{C}$. When these connections are sufficiently strong, the output of the spiny stellate subpopulation saturates, due to the nonlinear sigmoid function in Eq. (2). This nonlinearity has important consequences for event-related responses and the ensuing dynamics. In brief, the form of the impulse response function changes qualitatively with input
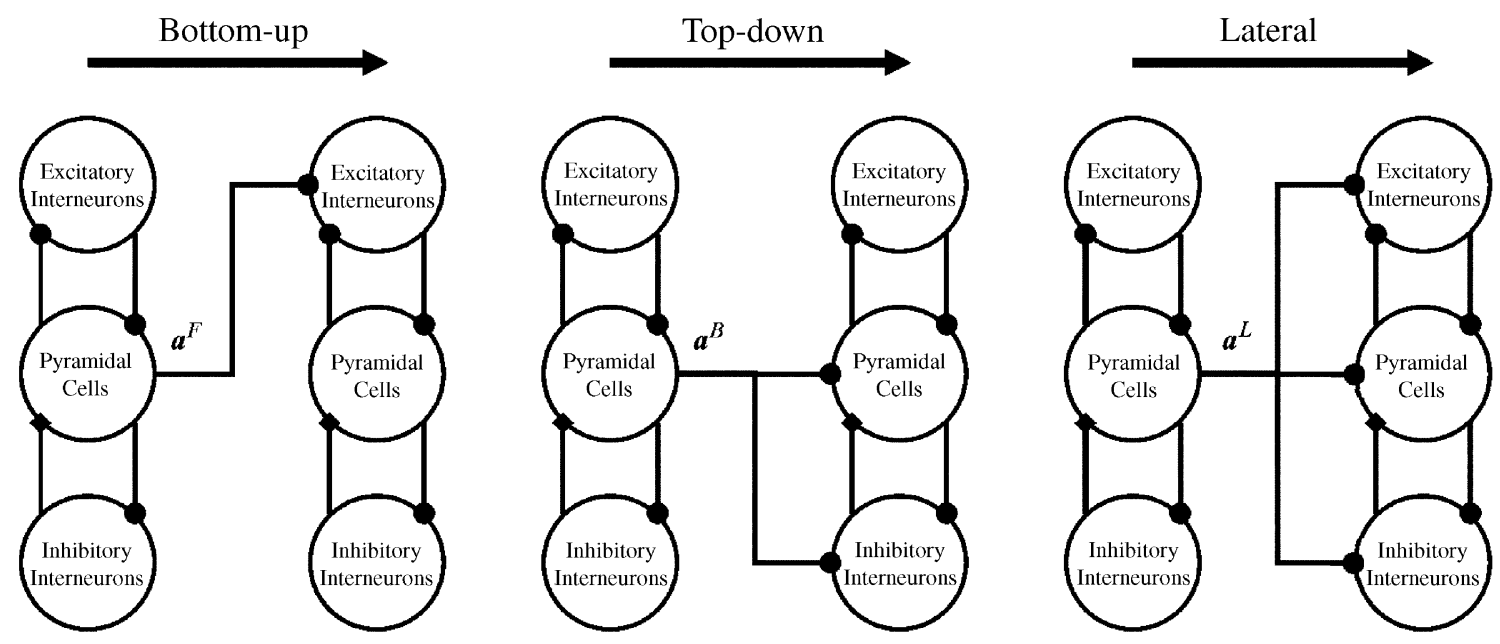

Fig. 3. Hierarchical connections among Jansen units (Fig. 2) based on simplified Felleman and van Essen rules (Fig. 1). Long-range connectivity is mediated by pyramidal cells axons. Their targets depend upon the type of connections. Coupling or connectivity parameters control the strength of each type of connection: $a^{\mathrm{F}}$ for forward, $a^{\mathrm{B}}$ for backward and $a^{\mathrm{L}}$ for lateral. 

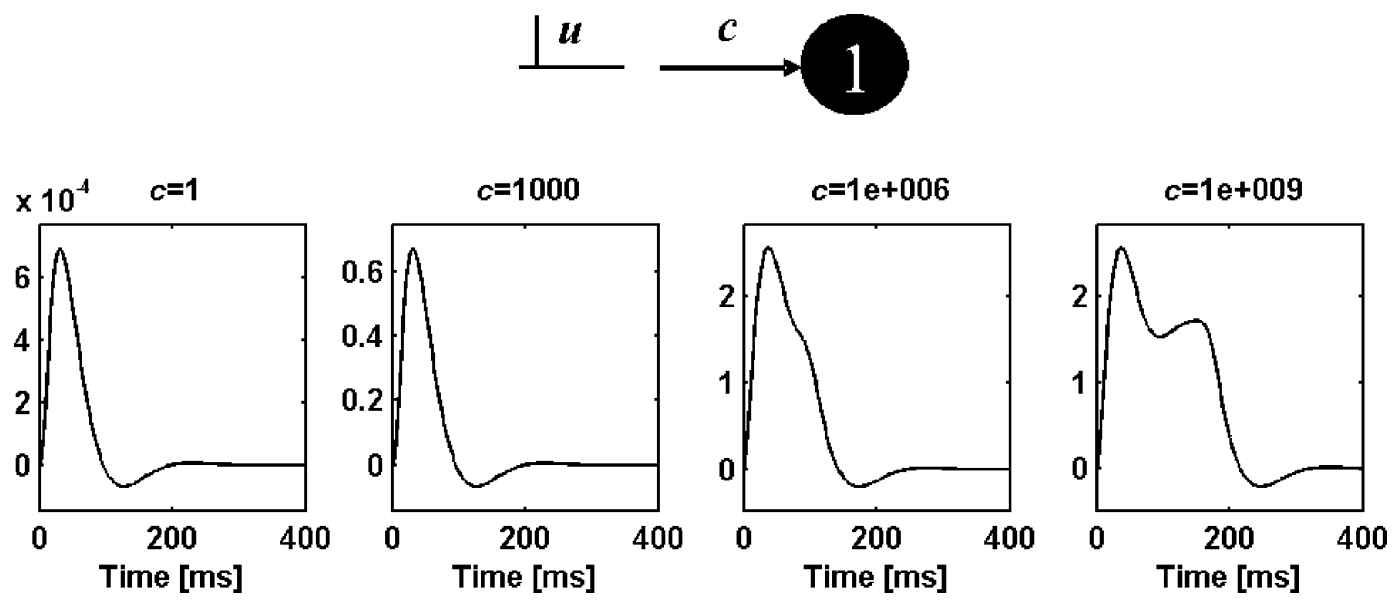

Fig. 4. The strength of input modulates the shape of MEG/EEG signal. The output of one area (variable $y$, see Appendix A) has been calculated for different values of $c$, the strength of forward connections mediating input $u$ (delta function). When $c$ is small $(c=1, c=1000)$, the output is not saturated and the MEG/ EEG signal $(y)$ amplitude is linearly related to $c$. For large values of $c\left(c=10^{6}, c=10^{9}\right)$, spiny stellate cells saturate and the shape of event-related MEG/EEG response changes substantially.

strength. To illustrate this point, we modelled a single area, which received an impulse at time zero and calculated the corresponding response for different values of $c$ (Fig. 4). With weak inputs, the response is linear, leading to a linear relationship between $c$ and peak MEG/EEG responses. However, with large values of $c$ neuronal activity leaving the linear domain of the sigmoid function in Eq. (2), the spiking saturates and the shape of the evoked response changes.

This behaviour is not surprising and simply reflects the nonlinear relationship between firing rates and post-synaptic depolarisation modelled by the nonlinearity. This nonlinearity causes saturation in responses of units to intrinsic and extrinsic inputs. For example, when the input is strong enough to saturate spiny stellate spiking, the pyramidal response exhibits a short plateau (right panel in Fig. 4). This saturation persists until the membrane potential of spiny stellate cells returns to its resting state. The sigmoid function models phenomenon; (i) at the single unit level, like refractoriness and spike rate adaptation and (ii) aspects of neuronal ensembles at the population level, like the distribution of thresholds involved in the generation of action potentials. The ensuing behaviour confers an inherent stability on dynamics because it is recapitulated in response to all bottom-up influences, as shown next.

\section{Bottom-up effects}

The targets of the forward connections and extrinsic inputs are identical. Therefore, the effects of $c$ and $a^{\mathrm{F}}$ on event-related responses, are exactly the same. Fig. 5 shows the simplest case of two areas (area 1 drives area 2). The difference, in relation to the previous configuration, is that area 1 has a gating effect. This is basically a low-pass filter, which leads to greater variation of the response in area 2, relative to responses elicited by direct input to area 2 (cf. Fig. 4). For instance, the small negative response component in area 1 , which follows the first positive deflection, is dramatically enhanced in area 2 for strong forward couplings. Again, this reflects the nonlinear behaviour of subpopulations responding to synaptic inputs.
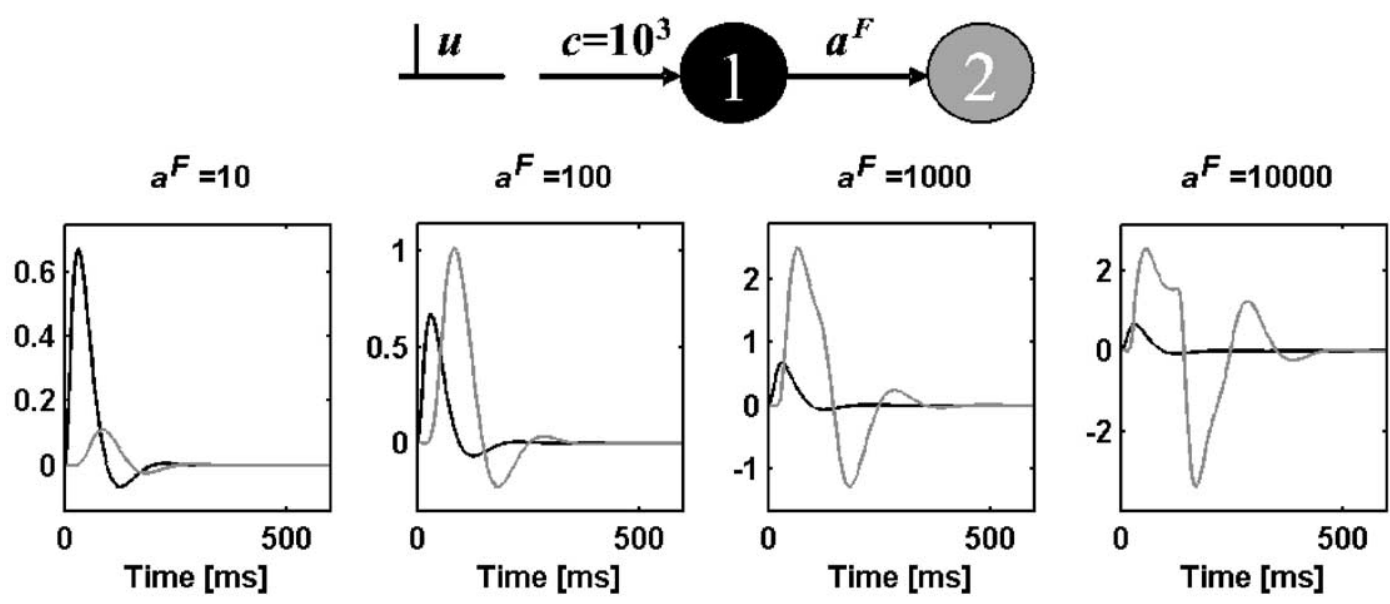

Fig. 5. The MEG/EEG signal of area 1 (black) and area 2 (grey) is plotted as a function of the forward connectivity $a^{\mathrm{F}}$. Bottom-up connectivity has the same effect as input connectivity $c$ : high values cause a saturation of spiny stellate cells (input cells), with a dramatic effect on MEG/EEG event-related responses. Nonlinear effects are particularly strong for the largest value of $a^{\mathrm{F}}$ (right panel) as the small negative component of area 1 (seen best in the left panel) induces a huge negative response in area 2 . 

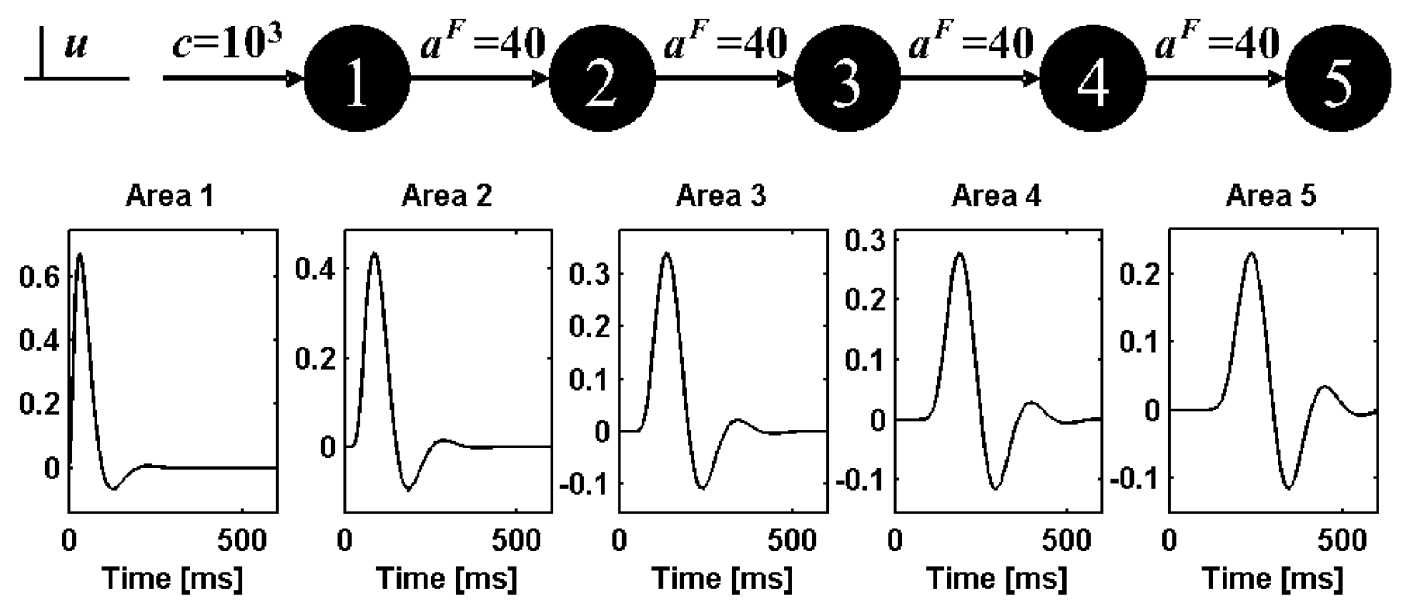

Fig. 6. A feedforward system composed of five areas. The MEG/EEG signal ( $y$ ) of each area elicited by a single pulse on area 1 is plotted in successive panels from left to right. Event-related activity lasts longer in high-level cortical areas of feedforward architectures. At each level in the hierarchy, the event-related response of pyramidal cells experiences successive low-pass filters, embodied by synaptic processes that transform the input signals to output.

\section{Level in hierarchy}

As mentioned above, activity is subject to low-pass filtering, by synaptic processes, each time it encounters a cortical region. A simple and intuitive consequence of this is that the form of eventrelated responses changes with each successive convolution in the hierarchy. To illustrate this point, we consider a feedforward configuration composed of five regions (Fig. 6). We see in Fig. 6 that, in addition to the propagation lag that delays the waveform at each level, the event-related response is more enduring and dispersed in higher-level areas. A useful heuristic here is that late components of evoked responses may reflect hierarchical processing at a deeper level. This effect is independent of synaptic time constants and connectivity parameters.

This simple delay and dispersion is not necessarily seen with more realistic configurations that involve top-down effects. In this context, late response components in higher cortical areas can reenter (Edelman, 1993) lower levels engendering complicated and realistic impulse response functions. In the reminder of this section, we look at the effects of adding backward and then lateral connections to the forward architecture considered above.

\section{Top-down effects}

Top-down connections mediate influences from high to lowlevel regions. Incoming sensory information is promulgated through the hierarchy via forward, and possibly lateral, connections to high-level areas. To demonstrate the effect of backward connections on MEG/EEG, we will consider a minimal configuration composed of two areas (Fig. 7). The fact that the forward and backward connections are different renders this functionally asymmetric architecture hierarchical. Although asymmetric, the presence of forward and backward connections creates loops. This induces stability issues as shown in Fig. 7: when backward connections are made stronger, damped oscillations $\left(a^{\mathrm{B}}=1 ; a^{\mathrm{B}}=10\right)$ are transformed into oscillations which ultimately stabilise $\left(a^{\mathrm{B}}=50\right)$ because of the saturation described in the previous subsection. Therefore, with $a^{\mathrm{B}}=50$, the stable attractor is a limit cycle and the resting state point attractor looses its dynamic stability. The dependence of oscillations on layers, loops and propagation delays has been the subject of much study in computational models (Lumer et al., 1997).
From a neurobiological perceptive, the most interesting behaviours are shown just prior to this phase-transition ${ }^{1}$ when damped oscillations are evident. Note that the peaks of the evoked response, in this domain, occur every $100 \mathrm{~ms}$ or so. This emulates the expression of late components seen empirically, such as the $\mathrm{N} 300$ or P400. The key point here is that late components, in the EEG/MEG, may reflect reentrant effects mediated by backward connections in hierarchical architectures. This observation fits comfortably with the notion that late EEG/MEG components reflect endogenous processing and depend explicitly on top-down effects. In short, late components may depend on backward connections and reflect a reentry of dynamics to hierarchically lower processing areas. This dependency can be seen clearly by comparing the two lefthand panels in Fig. 7 that show the emergence of late components on increasing the backward connection from one to ten.

The phase-transition from damped late components to oscillations is critical. Before the transition, the system is controllable. This means that the response can be determined analytically given the input. As discussed in Friston (2000a), long impulse responses endow the brain with a 'memory' of past inputs that enables perceptual processing of temporally extended events. In Friston (2000b), this was demonstrated using a Volterra kernel formulation and the simulation of spatio-temporal receptive fields in the visual system. However, after the transition it is no longer possible to determine when the input occurred given the output. This violates the principle of maximum information

\footnotetext{
${ }^{1}$ A phase-transition refers to the qualitative change in the systems attractor caused by changes in the systems parameters, here the coupling parameters. In the present context, increasing the backward coupling causes the point attractor to loose its dynamic stability (stability under small perturbations) and the emergence of a limit-cycle attractor. The nature of the phase-transition is usually assessed in terms of Lyapunov exponents (eigenvalues of the systems Jacobian $\partial f / \partial x$ ). When the system has a point attractor, the imaginary part of the principal or largest exponent is 0 . A limit cycle has nonzero imaginary parts and chaotic attractors have at least one real positive exponent. We do not present a stability analysis or the Lyapunov exponents in this work, because the phase-transitions are selfevident in the trajectories of the system.
} 

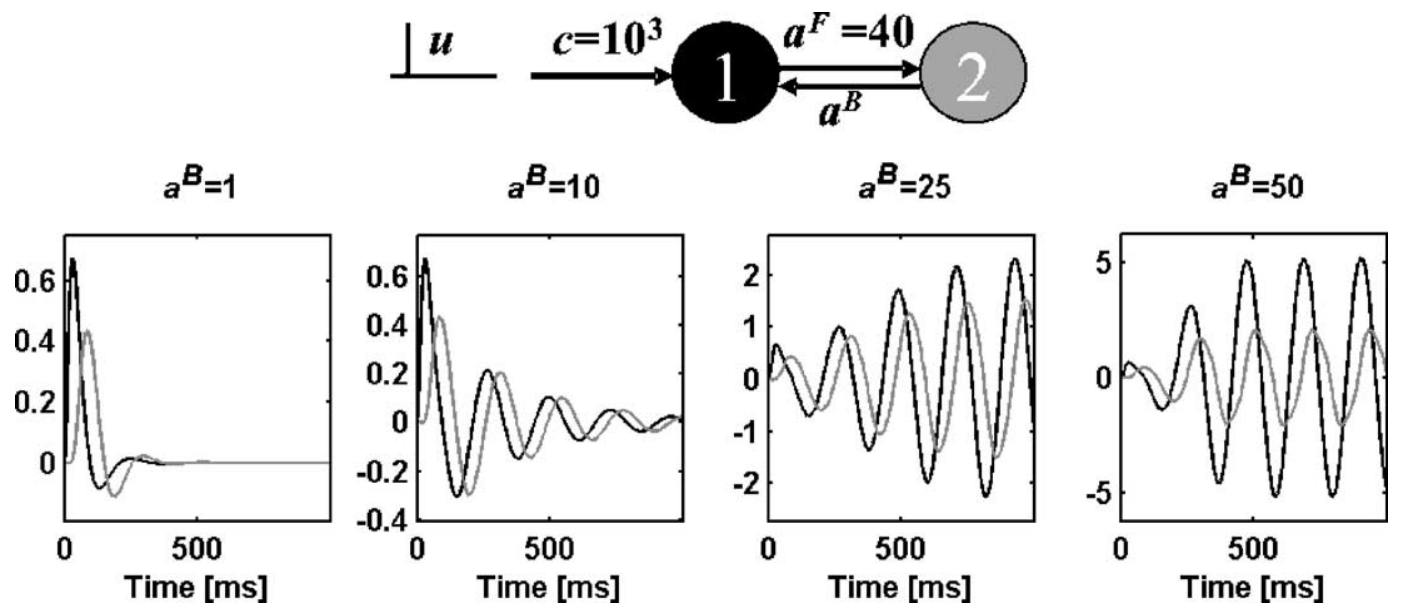

Fig. 7. Backward connections have a key influence on the stability of MEG/EEG event-related activity as demonstrated by this simple model composed of two areas (area coded in black and area 2 coded in grey). The forward connectivity $a^{\mathrm{F}}$ has been fixed to 40 and backward connectivity $a^{\mathrm{B}}$ varies between 1 and 50 from left to right. When top-down effects are small, their reentry leads to longer lasting event-related responses characterised by damped oscillations $\left(a^{\mathrm{B}}=1\right.$; $a^{\mathrm{B}}=10$ ). However, over a critical threshold of $a^{\mathrm{B}}$ (which depends upon $a^{\mathrm{F}}$ ), the system undergoes a phase-transition, loses its point attractor and expresses oscillatory dynamics $\left(a^{\mathrm{B}}=25 ; a^{\mathrm{B}}=50\right)$.

transfer (Linsker, 1990) and precludes this sort of response in the brain. In short, it is likely that reentrant dynamics prolong neuronal transients but will stop short of incurring a phasetransition to oscillations. If this phase-transition occurs, it is likely to be short-lived or pathological (e.g., photosensitive seizure activity).

It should be noted that the oscillations in the right-hand panels of Fig. 7 do not represent a mechanism for induced oscillations. The oscillations here are deterministic component of the systems impulse response function and are time-locked to the stimulus. Induced oscillations, by definition, are not time-locked to the stimulus and probably arise from a stimulusrelated change in the system's control parameters (i.e., shortterm changes in connectivity). We will return to this point later.

\section{Lateral connections}

Lateral connections link different regions at the same level in the hierarchy. They can be unidirectional or bi-directional as shown for the model in Fig. 8 with two areas. The main difference between forward and unidirectional lateral connections is that the latter target pyramidal cells. This means that the MEG/EEG signal is not so constrained by nonlinear saturation in layer 4 units. Therefore, as shown in Fig. 8a, the event-related response does not saturate for strong lateral connectivity values $a^{\mathrm{L}}$. On the other hand, bilateral connections and hierarchical connections are differentiated by the fact that bilateral connections are completely symmetric, which enable them to create a synchronisation manifold (Breakspear, 2002; Breakspear and Terry, 2002). A comparison of Fig. 8b and Fig. 7 shows that a special aspect of bilateral connections is their ability to support dynamics that are in phase. This sort of zero-lag phase-synchronisation is commonplace in the brain. Its mediation by lateral connections in this model concurs with previous modelling studies of zero-lag coupling in triplets of cortical areas that involve at least one set of bilateral or reciprocal connections (Chawla et al., 2001). For very large values of $a^{\mathrm{L}}$, architectures with bilateral connections are highly nonlinear and eventually undergo a second phase-transition (see Fig. 8b).
In this section, we have provided a deterministic characterisation of simple hierarchical models in terms of their impulse responses. We have tried to show that the model exhibits a degree of face validity in relation to real evoked responses and have related certain mechanistic aspects to previous modelling work to provide some construct validity. We now turn to the secondary biological focus of this paper; namely the plausibility of nonlinear mechanisms that might explain ERP/ERF components.

\section{Ongoing and event-related activity}

So far, we have considered noise-free systems. Event-related responses were modelled in terms of deterministic impulse responses that were unique to a given neuronal configuration. In this context, it is not necessary to evoke the notion of averaging. However, real MEG/EEG signals show a great variability from trial to trial (Arieli et al., 1996). In this section, we model this variability by adding a stochastic component (a zero-mean Gaussian process) to the input $u$. The output corresponding to a stochastic input is referred to ongoing activity, i.e. oscillations in the MEG/EEG signal that share no phase relationship with the stimulus. This does not mean that ongoing activity should be considered as noise, just that there is no obvious stimulus-related structure.

\section{Ongoing activity}

Ongoing activity is shaped by the same nonlinear convolution experienced by deterministic inputs. In the context of stationary inputs, the outputs can be characterised in terms of their spectral properties, which are determined by the generalised transfer functions of the Volterra kernels associated with any controllable analytic system. The impulse response function is the first-order kernel. As soon as the connectivity parameters of a hierarchical network change, the principal modes of this network, defined by the principal frequencies of oscillations, are modulated (David and Friston, 2003). As an illustration, let us consider the simple hierarchical model of two cortical areas established in the previous 
(a)
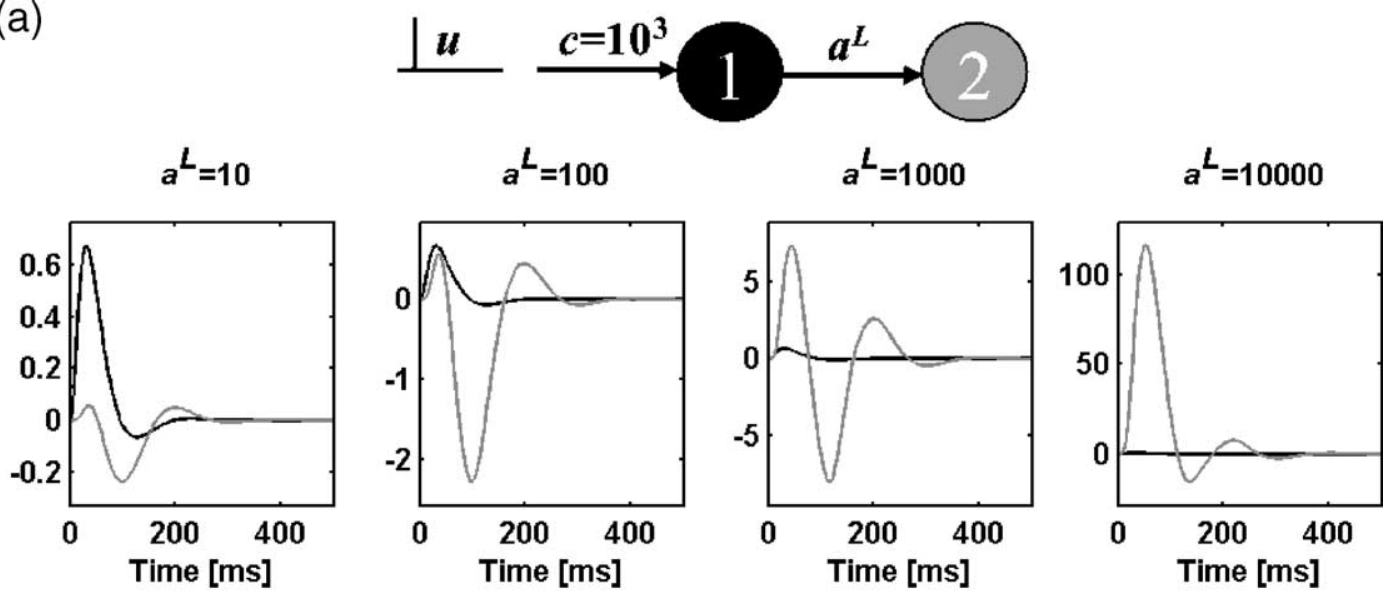

(b)
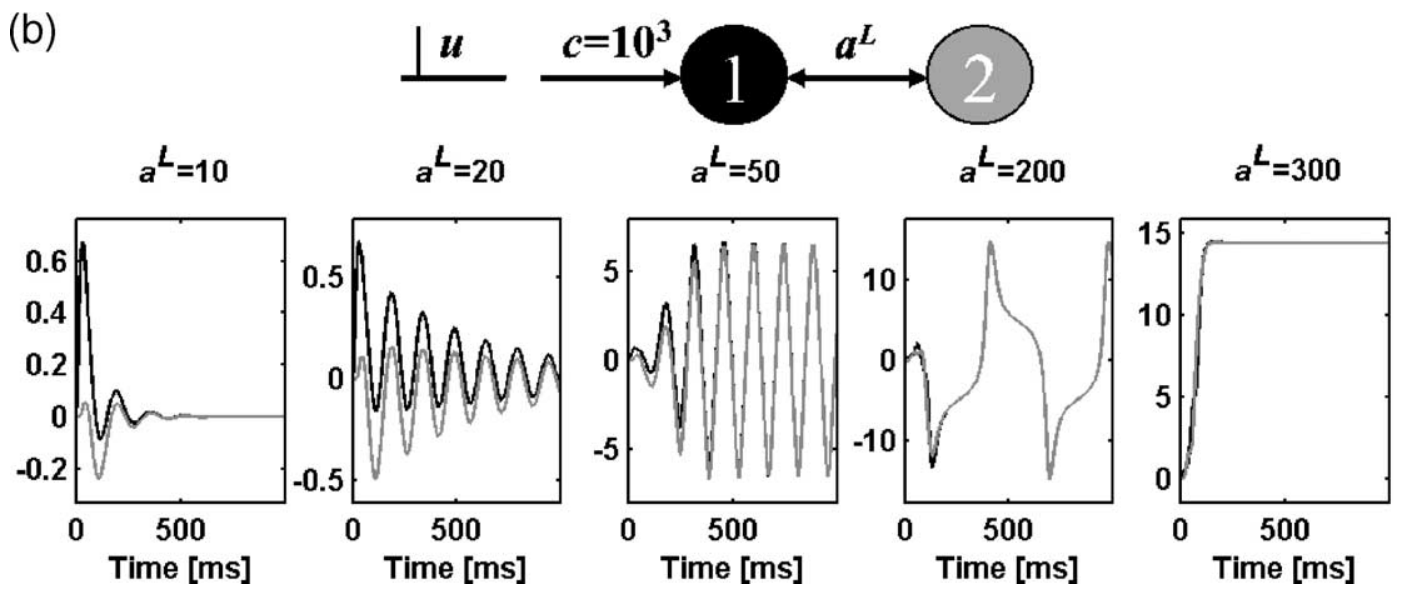

Fig. 8. The effects of lateral connections are shown with a simple model composed of two areas (black: area 1, grey: area 2). The depolarisation of pyramidal cells $(y)$ is plotted for several values of lateral connectivity $a^{\mathrm{L}}$. (a) Unilateral connections support transients that differ from those elicited by forward connections (Fig. 4). In particular, the saturation of layer 4 is not so important and the signal exhibits less saturation for large $a^{\mathrm{L}}$. (b) Increasing bi-directional lateral connections has a similar effect to increasing backward connections. The main difference is the relative phase of evoked oscillations, which are synchronised at zero-lag. For very large values of $a^{\mathrm{L}}$, the model is highly nonlinear and eventually exits the oscillatory domain of parameter space.

section (Fig. 9) with two configurations, which differ in the strength of backward connections $\left(a^{\mathrm{B}}=1\right.$ and $\left.a^{\mathrm{B}}=10\right)$. The corresponding frequency spectra, of pyramidal cell depolarisation of the two areas, show that the change in connectivity induces a profound modulation of their spectral profile. As one might intuit, increasing backward connections induce a peak at the same
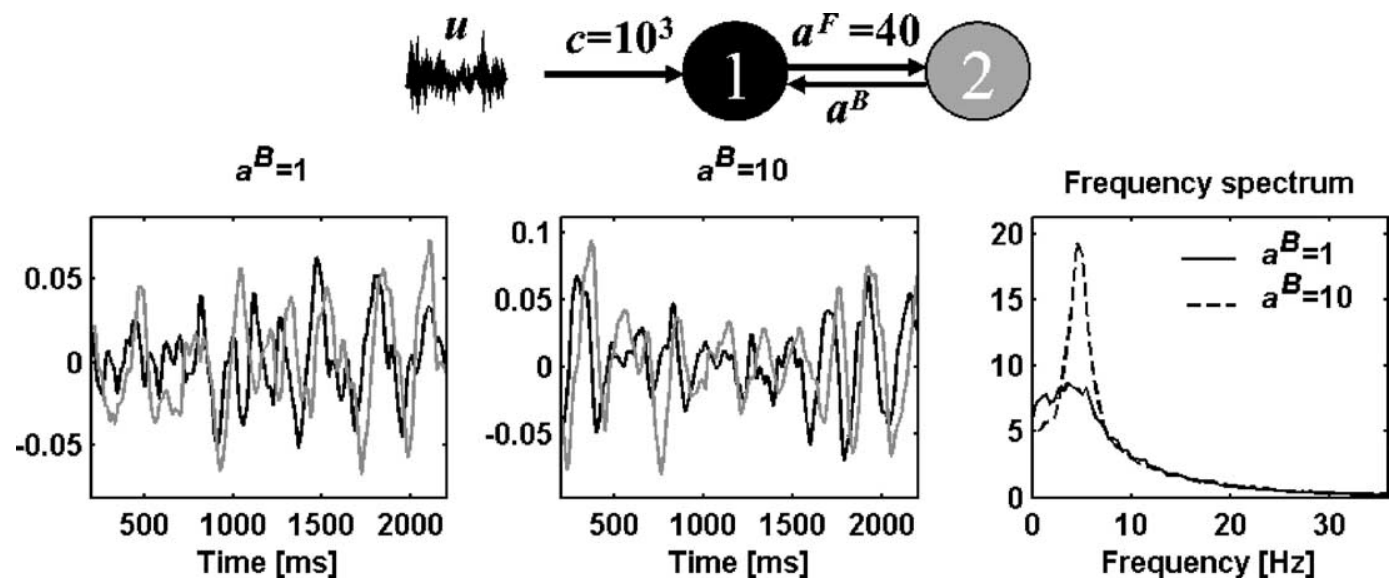

Fig. 9. The modulation of backward connectivity $\left(a^{\mathrm{B}}=1\right.$ or $\left.a^{\mathrm{B}}=10\right)$ has huge effect on the power spectrum of ongoing MEG/EEG dynamics (variable $y$ is plotted in black for area 1 and in grey for area 2). When $a^{\mathrm{B}}$ increases from 1 to 10 , there is loss of power below $3 \mathrm{~Hz}$, and an excess between 3 and $7 \mathrm{~Hz}$. The amplitude spectra in the right panel were obtained by averaging the modulus of the Fast Fourier Transform of pyramidal cell depolarisation, over 100 epochs of $2.5 \mathrm{~s}$ (examples are shown in the two left-hand panels, black: first area, grey: second area). 
frequency of the damped oscillations in the impulse response function. This is an important aspect of ongoing activity in the sense that its spectral behaviour may be very close to that of evoked transients as shown in Makeig et al. (2002).

\section{Induced vs. evoked dynamics}

This modulation of oscillatory dynamics, by the systems coupling parameters, provides a natural model for event-related changes in rhythmic activity. This phenomenon is known as eventrelated synchronisation (ERS) in frequency bands showing an evoked increase in power, or conversely, event-related desynchronisation (ERD) for decreases (Basar, 1980; Pfurtscheller and Lopes da Silva, 1999). In light of the above connectivity-dependent changes in power, ERD and ERS may reflect the dynamics induced by evoked changes in short-term plasticity. The key difference between evoked and induced transients relates to the presence or absence of changes in the systems control parameters, here connectivity or synaptic efficacy. Evoked changes are not necessarily associated with parameter changes and any complicated response can be ascribed to transients that arise as the systems trajectory returns to its attractor. Conversely, induced responses arise from perturbation of the attractor manifold itself, by changes in the parameters and ensuing changes in the dynamics. This distinction was discussed in Friston (1997) in relation to MEG dynamics and modelled using asymmetric connections between two areas in Friston (2000a).

Empirically, the ERS/ERD approach is used to look for MEG/ EEG power changes of rhythmic activity induced by external events. This phenomenon has been modelled, in the case of alpha rhythms, by a computational model of thalamo-cortical networks (Suffczynski et al., 2001). It has been shown that a key mechanism is the modulation of functional interaction between populations of thalamo-cortical cells and the reticular nucleus. This and related issues will be addressed in a subsequent paper on induced responses. Here, we focus on evoked changes.

The present analysis concludes by examining the sensitivity of evoked transients to state changes caused by ongoing activity, when the parameters are held constant. In particular, we see if this sensitivity can be expressed as phase-resetting. The key issue here is the presence of nonlinear interactions between the current state of the system and its perturbation by a stimulus.

\section{Phase-resetting and nonlinear interactions}

In the following, we investigate the effect of ongoing activity, on stimulus-dependent responses, to reconcile apparently contradictory conclusions from studies of event-related potentials. On one hand, classical studies have shown that event-related potentials are associated with amplitude changes in the MEG/EEG signal that represent a linear summation of an impulse response and ongoing activity (Arieli et al., 1996; Shah et al., 2004). In this scheme, the variability at the single-trial level is due to, and only to, ongoing activity, which is removed after averaging to estimate the impulse response. On the other hand, it has been hypothesised that eventrelated waveforms, obtained after averaging, could be due to a phase-resetting of ongoing activity with no necessary change in the amplitude (i.e., power) of any stimulus locked transient (Jansen et al., 2003; Makeig et al., 2002). Although mathematically well defined, the neural mechanisms that could instantiate phaseresetting of ongoing activity are unknown.
We will take phase-resetting to imply a nonlinear interaction between ongoing activity and stimulus-related input that results in phase-locking to stimulus onset. Although phase-locking can be produced by evoking oscillatory transients (i.e., amplitude modulation), this mechanism involves no change or resetting of the ongoing dynamics. To assess the contribution of phaseresetting in our simulations, we therefore need to look for interactions between ongoing and stimulus-related inputs that produced phase-locking in the outputs. As mentioned in the Introduction, this was addressed, in a simple way, by subtracting the response to ongoing activity alone from the response to a mixture of ongoing activity and stimulus input. In the absence of interactions, this difference (the evoked response) should be the same. On the other hand, if interactions are prevalent, the difference should change with each realisation of ongoing activity. We performed these analyses with different levels of input and assessed the degree of phase-locking in the outputs with the phase-locking value (PLV) (Lachaux et al., 1999; Tallon-Baudry et al., 1996): $\operatorname{PLV}(t)=\left|\langle\exp (j \phi(t))\rangle_{\text {trials }}\right|$ where the instantaneous phase $\phi(t)$ was obtained from the Hilbert transform (Le Van Quyen et al., 2001).

\section{Testing for interactions}

To evaluate the effect of background activity on single-trial event-related responses, we used the two area hierarchical model above, with $a^{\mathrm{B}}=1$ (Fig. 9). The first area was driven by an impulse function (stimulus) and Gaussian random noise (background activity) of standard deviation $\sigma=0.05$. The output of this region can be considered a mixture of evoked response and ongoing activity. We considered two conditions: one with low levels of mixed input $\left(c=10^{2}\right)$ and another with high levels $\left(c=2 \cdot 10^{4}\right)$. These values were chosen to emphasise the systems nonlinear properties; with the smaller value of $c$, neuronal responses remain largely to the linear regime of the nonlinear function. The larger value of $c$ was chosen so that excursions of the states encroached on the nonlinear regime, to produce neuronal saturation in some trials. In both cases, the stimulus was a delta-function. The simulated responses, for 100 trials, are shown in Fig. 10.

When input levels are low (left hand side of Fig. 10), eventrelated activity at the single-trial level shows a relatively reproducible waveform after stimulus onset (Fig. 10B). This transient is reflected in the ERP/ERF after averaging (Fig. 10C). To confirm the experimental results of Arieli et al. (1996), we decomposed each event-related response into two components. First, the stochastic component (the response to ongoing activity alone-Fig. 10D) and second, an extra component elicited by adding the stimulus (Fig. 10E). This is the difference between the response elicited by stochastic component alone (Fig. 10D) and the response to the mixed input (Fig. 10B). If the system was linear, these differences should not exhibit any variability over trials, and thus define the "reproducible response" (Arieli et al., 1996). Effectively, the stimulus-dependent component shows no variability and we can conclude that the response components due to stimulus and ongoing activity are linearly separable. In other words, there are no interactions that could mediate phase-resetting. Despite this, there is ample evidence for phase-locking. This is shown in Fig. 10F, using the PLV index.

However, the situation is very different when we repeat the simulations with high input levels (right hand side of Fig. 10). In this context, the event-related responses do not show any obvious 

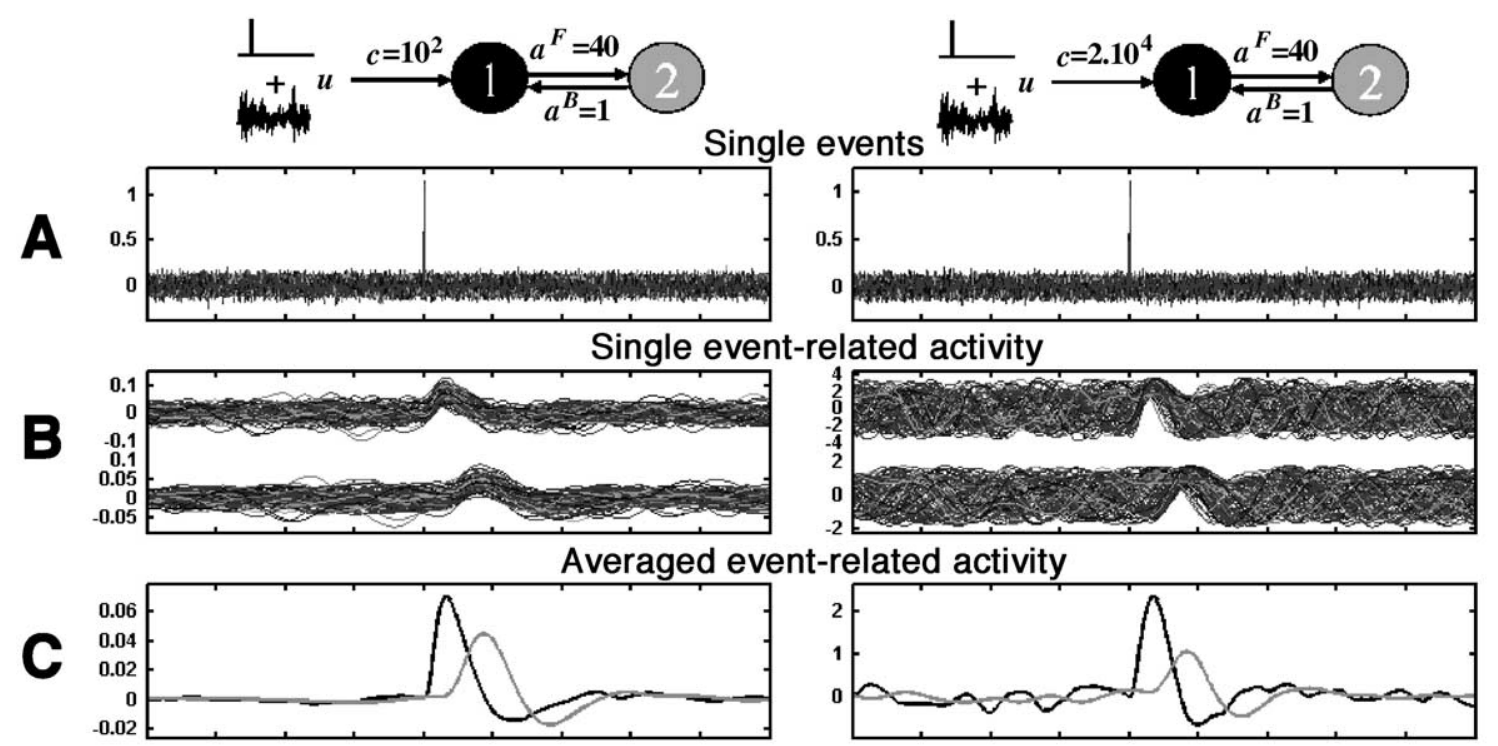

Single ongoing activity
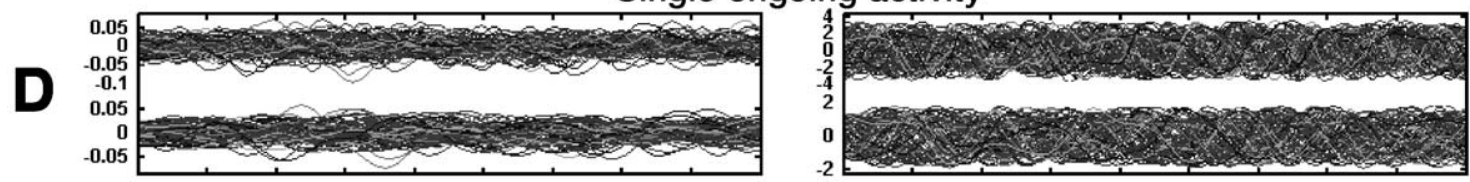

Linear estimation of single event-related activity
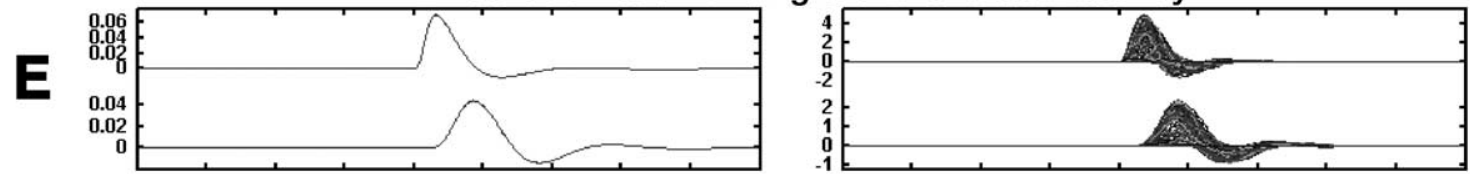

Phase-locking value
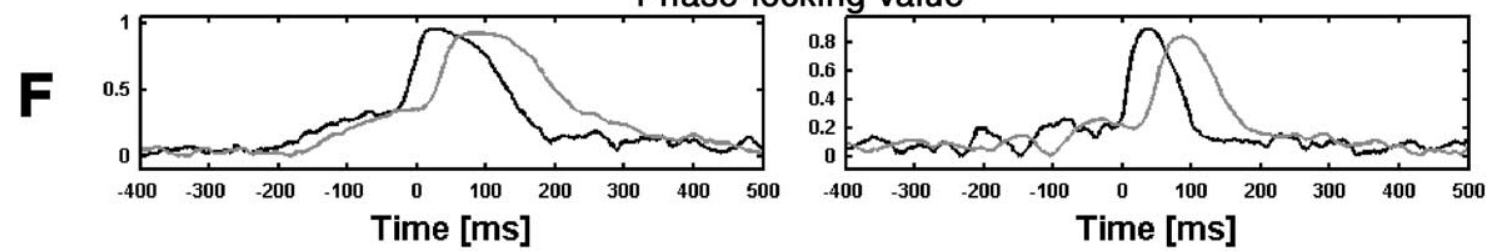

Fig. 10. Event-related responses in the context of ongoing activity (100 trials). Two hierarchically connected regions are considered. Input (ongoing and stimulus-related) enters into the system through region 1. Two levels of input are considered: weak on the left-hand side $\left(c=10^{2}\right)$, strong on the right-hand side $\left(c=2.10^{4}\right)$. The successive horizontal panels show different types of activity. The time scale is identical for each panel and shown at the bottom. (A) Inputs, comprising a delta function and Gaussian noise of standard deviation 0.05 (stimulus onset at time 0 ). (B) Event-related activity ( $y$ ) at the single-trial level. The time series over trials is shown (area 1 is above area 2). (C) Averaged event-related response estimated by averaging over epochs shown in panel (B) (area 1 in black, area 2 in grey). (D) Responses ( $y$ ) to the noisy input without the delta function, shown in the same format as in panel (B). (E) Stimulus-dependent component obtained from subtracting panel (D) from panel (B). (F) Phase-locking value computed form time series in panel (B), which exhibits a transient phase-synchronisation to peristimulus time (black: area 1, grey: area 2).

increase in amplitude after the stimulus (Fig. 10B). However, the averaged event-related activity (Fig. 10C) is very similar to that above (left hand side of Fig. 10C). The fact that one obtains an ERP by averaging in this way suggests that the stimulus input induced phase-resetting of the ongoing oscillations. This is confirmed by the large variation in stimulus-dependent components from trial to trial. This variation reflects nonlinear interactions between the stimulus and ongoing activity (Fig. 10E). These interactions are associated with phase-locking as shown in Fig. 10F.

In summary, the fact that the difference in evoked responses with and without background noise (panel e, Fig. 10) shows so much variability, suggests that background activity interacts with the stimulus: When ongoing activity is high, stellate cells outputs saturate and the stimulus-related response is attenuated. Conversely, when ongoing activity is low the evoked-response is expressed fully. This dependency on ongoing activity is revealed by variation in the evoked responses with high input levels. In conclusion, the apparently contradictory results presented in Arieli et al. (1996), Jansen et al. (2003), Makeig et al. (2002) and Shah et al. (2004) can be reproduced in most part and reconciled within the same framework. With high activity levels, the ongoing and stimulusdependent components interact, through nonlinearities in the population dynamics, to produce phase-resetting and a classical ERP on averaging. When activity is lower, the stimulus and endogenous dynamics do not interact and the ERP simply reflects the transient evoked by stimuli that is linearly separable from ongoing dynamics. 


\section{Discussion}

We have shown that it is possible to construct hierarchical models for MEG/EEG signals. To that end, we have assumed an architecture for cortical regions and their connections. In particular, we have used the Jansen model (Jansen and Rit, 1995) for each source, and a simplified version of the connection rules of Felleman and Van Essen (1991) to couple these sources. Here, we have fixed the parameters intrinsic to each source (synaptic time constants, output function and intrinsic connections) and have focused on manipulating extrinsic connections among areas. We have used this model to address some issues in the genesis of evoked responses as observed with MEG/EEG.

Inferring neural mass models and their parameters, on the basis of the EEG and MEG alone, is a dynamical inverse problem that does not have a unique solution. The model we have described is one possible model among many others. We have used this model to afford a balance between simple neuronal models that are unrealistic but sufficient to emulate dynamics seen empirically, and more complicated models that have an explicit relation to electrophysiology but are difficult to manipulate. In a future work, we will compute the relative likelihood of, or evidence for, different models (with Bayesian model selection) using real MEG/EEG data (David et al., 2004b). At this stage, we focus on establishing the face validity of a representative model by showing that it can reproduce a range of biological behaviours.

\section{Deterministic simulations}

In Section 3, we ignored the stochastic component of MEG/ EEG signals. This allowed us to study various configurations in terms of their deterministic input-output behaviour. Each type of extrinsic connection (forward, backward and lateral) had specific effects on event-related dynamics. The key conclusions from these analyses were: (i) When forward connections, mediating bottom-up or extrinsic inputs, are sufficiently strong, nonlinear mechanisms cause a saturation of neuronal responses. This endows the system with an inherent stability that precludes nondissipative dynamics. (ii) The duration of evoked transients increases with the hierarchical depth or level of processing. (iii) When backward or bilateral connections are added, evoked transients become more protracted, exhibiting damped oscillations. These are formally identical to late or endogenous components.

These simulations suggest that late components are mediated by reentrant dynamics within cortical hierarchies. Increasing the strength of extrinsic backward or bilateral connections causes the number of late components to increase until a phase-transition into a quasi-oscillatory regime, whose dynamic stability is assured by the nonlinear saturation above. We discussed, briefly, the importance of this phase-transition in relation to information theoretic constraints on neuronal computations. In short, it is likely that selective pressure will extend the duration of transients so that the mutual information between current activity and past perceptual brain states is maximised (see Friston, 2000a for a fuller discussion). However, phase-transitions to oscillation are not adaptive because the system is no longer controllable and the informational link with the past is lost. A simple mechanism for increasing the memory of neuronal systems is to re-enter past states from hierarchical levels via backward connections. This is precisely what we simulated. In summary, late components may represent dynamic "echoes" that allow current sensory information to interact with perpetual constructs from higher areas, based on previous sensory input. We have discussed one form of this hierarchical reentry, using an empirical Bayesian perspective on predictive coding (Friston, 2002).

\section{Stochastic simulations}

In the second section, we introduced stochastic components to the inputs, to study event-related responses in the context of background activity. This work was motivated by apparently contradictory views of ERP/ERF generation. Some authors (Arieli et al., 1996; Shah et al., 2004) have argued that ERPs/ERFs can be considered as the impulse response function of a linear system, despite the huge variability at the single-trial level. In contrast, it has been proposed (Duzel et al., 2003; Jansen et al., 2003; Klimesch et al., 2004; Makeig et al., 2002) that ERPs/ERFs could be due to a phase-resetting process that does not require the notion of a linear impulse response. This induces some hypothetical neural mechanism that can implement a phase-resetting of ongoing activity. These two views are supported by analyses of real MEG/ EEG data. We showed that our model reproduces data that support both views. In our framework, the key factor is the level of activity. When activity is low, the model operates in a quasi-linear regime and reproduces the linear behaviours reported in Arieli et al. (1996). Conversely, when activity is high, single-trial responses do not show any amplitude modulation and yet still produce an ERP on averaging. This is consistent with the observations of Jansen et al. (2003) and Makeig et al. (2002).

Our analyses suggest that phase-resetting involves a nonlinear interaction between stimulus-related responses and ongoing activity. This observation is very reminiscent of a similar finding, pertaining to fast-oscillatory dynamics induced in simulated populations. These simulations (Chawla et al., 2000) showed that functional connectivity or dynamic integration between two populations increases with mean background activity and with stimulus-related rate modulation. Furthermore, as the background activity increases, the populations become increasingly sensitive to the intensity of the stimulus in terms of a predisposition to transient phase-locking. This reflected an interaction between background activity and stimulus-intensity in producing dynamic correlations. The mechanism of these interactions was modelled at the level of membrane time constants and temporal integration over milliseconds, using single-compartment units and the Hodgkin-Huxley formalism. Our simulations were at a much less detailed level, employing mean field-like approximations. Furthermore, they addressed evoked transients as opposed to induced fast dynamics. However, they both speak to the central role of nonlinear interactions between ongoing activity and stimuli in causing phase-locking. In our case, of evoked responses, this phase-locking was to stimulus onset and the mechanism can be attributed directly to the only nonlinearity in our model: namely the sigmoid response function transforming depolarisation to firing rate. This nonlinearly renders ongoing activity sensitive to stimulus perturbations (this is the definition of an interaction). This sensitivity is due to the saturating nature of the sigmoid function. When ongoing activity is high, the system states are close to the nonlinear regime of the sigmoid function and perturbations due to the stimulus cause neuronal saturation. This 
saturation causes the ongoing activity to "forget" its history and experience a resetting of its phase.

\section{Phase-resetting vs. phase-locking}

In this paper, we have made a clear distinction between phaselocking and phase-resetting. Phase-locking, as measured by the PLV implies a statistical dependency among phases, over ERPs (that is, knowing the phase from one ERP allows one to predict the phase of another). Phase-locking can be mediated in a number of ways. As shown in Fig. 10F, there is no real difference between the phase-locking measures obtained from linear and nonlinear singletrial responses. Conversely, phase-resetting refers to a change in the phase, within ERPs. This can be mediated by linear or nonlinear mechanisms (i.e., a second or high-order interaction between ongoing dynamics and the stimulus). The key debate here is not about phase-resetting itself, but whether it is caused by nonlinear mechanisms (i.e., without changes in amplitude). We therefore focused on the distinction between linear or nonlinear, rather than phase-resetting per se. In a future paper, we will look at phase-resetting, explicitly, using background activity that is periodic and of known phase (see below).

In this paper, phase-resetting is taken to imply a nonlinear interaction between ongoing dynamics and a stimulus that induces a phase-locking over trials. This generalises the notion of alpha phase-resetting that is usually characterised empirically. Our simulations and analysis were not limited to a single frequency. The ongoing activity we used was broad-spectrum and the phaselocking measure was not frequency-specific. In empirical studies, it is not possible to analyse the interaction between stimulus and ongoing activity, because no single trial can be replicated in the absence of the stimulus. This means that empirical studies have to use epochs that show oscillations at a particular frequency to demonstrate phase-resetting. In a future communication, we will apply this phase-resetting analysis (cf. (Makeig et al., 2002)) to stimulations in which our stochastic ongoing activity is replaced with a deterministic sinusoidal forcing term, in the alpha range. This will allow us to partition phase-locking into components that are mediated by linear superposition and phase-resetting respectively.

\section{Evoked vs. induced oscillations}

We have also noted that event-related activity is not restricted to ERPs/ERFs. It encompasses event-related changes in oscillations (power and synchronisation). We have suggested that this phenomenon can be explained by changes of connectivity due to short-term neuronal plasticity (see Friston, 2000a for a related discussion on nonlinear connections). We will be pursuing this in a subsequent paper on induced responses. The same changes in connectivity are also responsible for the modulation of long-range synchrony (or any type of interdependence measure) between distant MEG/EEG oscillators (David and Friston, 2003; David et al., 2004a).

\section{Modelling and estimation}

Generally, it was striking how different connections engendered such diverse event-related waveforms. This raises the issue of how models, of the sort described here, can be applied to real data. The long-term agenda of our modelling programme is to establish the validity of neuronal network models so that they can be used as forward models to explain real data. The key advantage of this approach is that the parameters of the model, which are estimated, have a direct physiological interpretation. Several studies have shown that it is possible to estimate the parameters of observation models using real MEG/EEG data, in the case of both rhythmic activity (Valdes et al., 1999) and ERPs (Rennie et al., 2002). An important issue, in this context, is the ability to constrain, or regularise, the inverse problem that dynamic forward models like this pose. These issues can be addressed using Bayesian inference and dynamic causal modelling (Friston et al., 2003). A great advantage of using neuronally plausible forward models is that constraints on the solution can be applied, in an informed way, using priors on the model parameters. At present, our team has published Bayesian inference procedures for dynamic causal modelling of functional MRI (Friston et al., 2003; Penny et al., 2004). In forthcoming reports, we will extend this approach to a variety of models, including the neural mass model for MEG/EEG described here. A preliminary report has already been published in which we estimate the parameters of this neural mass model but without localising the sources (David et al., 2004b). In our next communication, we will combine neural mass models and forward MEG/EEG modelling to study cognitive functions, and in particular to infer changes in connectivity among experimental conditions.

\section{Conclusion}

We have shown that neural mass models (David and Friston, 2003; Jansen and Rit, 1995; Lopes da Silva et al., 1997; Nunez, 1974; Rennie et al., 2002; Robinson et al., 2001; Stam et al., 1999; Suffczynski et al., 2001; Valdes et al., 1999; Wendling et al., 2002) can reproduce a large variety of MEG/EEG signal characteristics. The potential advantage they afford, in comparison to standard data analysis, is their ability to pinpoint specific neuronal mechanisms underlying normal or pathological activity. Effort is needed to incorporate them, more systematically, in MEG/EEG analyses to enable enquiry into mechanistic questions about macroscopic neuronal processes. In forthcoming studies, we will describe the estimation of such models in a Bayesian framework (Friston et al., 2002) and will apply the present model to the analysis of real ERPs.

\section{Acknowledgment}

This work was supported by the Wellcome Trust.

\section{Appendix A}

In this appendix, we provide differential equations for the $i^{\text {th }}$ of $l$ cortical areas of the hierarchical Jansen model as described in Section 2.3. The activity of the $i^{\text {th }}$ cortical area is described by eight state variables $x^{(i)}$, the rate of change of which is described by an equation of the following form:

$\dot{x}^{(i)}(t)=f\left(x^{(i)}(t), u^{(i)}(t), S\left(y^{(j)}\left(t-\delta^{(i j)}\right)\right), \mathbf{A}^{F}, \mathbf{A}^{B}, \mathbf{A}^{L}, \boldsymbol{C}\right)$

with $j \neq i$.

$u^{(i)}$ is the extrinsic input to region $i$ not modelled by other areas. $S\left(y^{(j)}\right)$ is the firing rate of pyramidal cells of region $j$. $\mathbf{A}^{\mathrm{F}}, \mathbf{A}^{\mathrm{B}}$ and $\mathbf{A}^{\mathrm{L}}$ are $l \times l$ connectivity matrices for forward, 
backward and lateral connections, respectively. $\boldsymbol{C}$ is the connectivity vector for extrinsic inputs. $\delta^{(i j)}$ is the propagation delay between region $i$ and region $j$. Eqs. (1) and (2) in the main text describe neuronal input and output operators that correspond to the following state equation which is the specific version of Eq. (A.1) for our model:

$$
\begin{aligned}
\dot{x}_{1}^{(i)}= & x_{4}^{(i)} \\
\dot{x}_{4}^{(i)}= & \frac{H_{e}}{\tau_{e}}\left(\sum_{\substack{j=1 \\
j \neq i}}^{l} \mathbf{A}^{F}(i, j) S\left(y^{(j)}\left(t-\delta^{(i j)}\right)\right)\right. \\
& \left.+\sum_{\substack{j=1 \\
j \neq i}}^{l} \mathbf{A}^{L}(i, j) S\left(y^{(j)}\left(t-\delta^{(i j)}\right)\right)+C(i) u^{(i)}+\gamma_{1} S\left(y^{(i)}\right)\right) \\
& -\frac{2}{\tau_{e}} x_{4}^{(i)}-\frac{x_{1}^{(i)}}{\tau_{e}^{2}} \\
\dot{x}_{2}^{(i)}= & x_{5}^{(i)} \\
\dot{x}_{5}^{(i)}= & \frac{H_{e}}{\tau_{e}}\left(\sum_{\substack{j=1 \\
j \neq i}}^{l} \mathbf{A}^{B}(i, j) S\left(y^{(j)}\left(t-\delta^{(i j)}\right)\right)\right. \\
& \left.+\sum_{\substack{j=1 \\
j \neq i}}^{l} \mathbf{A}^{L}(i, j) S\left(y^{(j)}\left(t-\delta^{(i j)}\right)\right)+\gamma_{2} S\left(x_{1}^{(i)}\right)\right) \\
& -\frac{2}{\tau_{e}} x_{5}^{(i)}-\frac{x_{2}^{(i)}}{\tau_{e}^{2}}
\end{aligned}
$$$$
\dot{x}_{3}^{(i)}=x_{6}^{(i)}
$$$$
\dot{x}_{6}^{(i)}=\frac{H_{i}}{\tau_{i}} \gamma_{4} S\left(x_{7}^{(i)}\right)-\frac{2}{\tau_{i}} x_{6}^{(i)}-\frac{x_{3}^{(i)}}{\tau_{i}^{2}}
$$$$
\dot{x}_{7}^{(i)}=x_{8}^{(i)}
$$

$$
\begin{aligned}
\dot{x}_{8}^{(i)}= & \frac{H_{e}}{\tau_{e}}\left(\sum_{\substack{j=1 \\
j \neq i}}^{l} \mathbf{A}^{B}(i, j) S\left(y^{(j)}\left(t-\delta^{(i j)}\right)\right)\right. \\
& \left.+\sum_{\substack{j=1 \\
j \neq i}}^{l} \mathbf{A}^{L}(i, j) S\left(y^{(j)}\left(t-\delta^{(i j)}\right)\right)+\gamma_{3} S\left(y^{(i)}\right)\right) \\
& -\frac{2}{\tau_{e}} x_{8}^{(i)}-\frac{x_{7}^{(i)}}{\tau_{e}^{2}}
\end{aligned}
$$

where $y^{(i)}=x_{2}^{(i)}-x_{3}^{(i)}$ is the pyramidal cells membrane potential (depolarisation) of region $i$. This is assumed to be proportional to the cortically reconstructed current source densities obtained from MEG/EEG scalp signals. This equation was integrated using standard Runge-Kutta techniques (Kloeden and Platen, 1999) with an integration time-step of $1 \mathrm{~ms}$. Apart from the extrinsic connectivity parameters, we used the same parameters for every simulation: $H_{e}=3.25, H_{i}=29.3, \tau_{e}=10 \mathrm{~ms}, \tau_{i}=15 \mathrm{~ms}, \delta=\delta^{(i j)}=$ $10 \mathrm{~ms}, \gamma_{1}=50, \gamma_{2}=40, \gamma_{3}=\gamma_{4}=12, e_{0}=2.5, v_{0}=0, r=0.56$.

\section{References}

Abeles, M., 1991. Corticonics: Neural Circuits of the Cerebral Cortex. Cambridge University Press, Cambridge.

Arieli, A., Sterkin, A., Grinvald, A., Aertsen, A., 1996. Dynamics of ongoing activity: explanation of the large variability in evoked cortical responses. Science 273, 1868-1871.

Baillet, S., Mosher, J.C., Leahy, R.M., 2001. Electromagnetic brain mapping. IEEE Signal Process. Mag., 14-30.

Basar, E., 1980. EEG-Brain Dynamics: Relation Between EEG and Brain Evoked Potentials. Elsevier, New York.

Breakspear, M., 2002. Nonlinear phase desynchronization in human electroencephalographic data. Hum. Brain Mapp. 15, 175-198.

Breakspear, M., Terry, J.R., 2002. Nonlinear interdependence in neural systems: motivation, theory, and relevance. Int. J. Neurosci. 112, $1263-1284$.

Breakspear, M., Terry, J.R., Friston, K.J., 2003. Modulation of excitatory synaptic coupling facilitates synchronization and complex dynamics in a biophysical model of neuronal dynamics. Network 14, 703-732.

Chawla, D., Lumer, E.D., Friston, K.J., 2000. Relating macroscopic measures of brain activity to fast, dynamic neuronal interactions. Neural Comput. 12, 2805-2821.

Chawla, D., Friston, K.J., Lumer, E.D., 2001. Zero-lag synchronous dynamics in triplets of interconnected cortical areas. Neural Netw. 14, $727-735$

Coles, M.G.H., Rugg, M.D., 1995. Event-related brain potentials: an introduction. In: Rugg, M.D., Coles, M.G.H. (Eds.), Electrophysiology of Mind. Oxford University Press, Oxford, pp. 1-26.

Crick, F., Koch, C., 1998. Constraints on cortical and thalamic projections: the no-strong-loops hypothesis. Nature 391, 245-250.

David, O., Friston, K.J., 2003. A neural mass model for MEG/EEG: coupling and neuronal dynamics. NeuroImage 20, 1743-1755.

David, O., Cosmelli, D., Friston, K.J., 2004a. Evaluation of different measures of functional connectivity using a neural mass model. NeuroImage 21, 659-673.

David, O., Harrison, L., Kilner, J., Penny, W., Friston, K.J., 2004b. Studying effective connectivity with a neural mass model of evoked MEG/EEG responses. In: Halgren, E., Ahlfors, S., Hamalainen, M., Cohen, D. (Eds.), Proceedings of the 14th international conference on biomagnetism BIOMAG 2004, pp. 135-138. Boston.

De Groff, D., Neelakanta, P.S., Sudhakar, R., Aalo, V., 1993. Stochastical aspects of neuronal dynamics: Fokker-Planck approach. Biol. Cybern. $69,155-164$.

DeFelipe, J., Alonso-Nanclares, L., Arellano, J.I., 2002. Microstructure of the neocortex: comparative aspects. J. Neurocytol. 31, 299-316.

Duzel, E., Habib, R., Schott, B., Schoenfeld, A., Lobaugh, N., McIntosh, A.R., Scholz, M., Heinze, H.J., 2003. A multivariate, spatiotemporal analysis of electromagnetic time-frequency data of recognition memory. NeuroImage 18, 185-197.

Edelman, G.M., 1993. Neural Darwinism: selection and reentrant signaling in higher brain function. Neuron 10, 115-125.

Engel, A.K., Fries, P., Singer, W., 2001. Dynamic predictions: oscillations and synchrony in top-down processing. Nat. Rev. Neurosci. 2, 704-716.

Felleman, D.J., Van Essen, D.C., 1991. Distributed hierarchical processing in the primate cerebral cortex. Cereb. Cortex 1, 1-47.

Fourcaud, N., Brunel, N., 2002. Dynamics of the firing probability of noisy integrate-and-fire neurons. Neural Comput. 14, 2057-2110.

Freeman, W.J., 1978. Models of the dynamics of neural populations. Electroencephalogr. Clin. Neurophysiol. 34, 9-18 (Suppl).

Friston, K.J., 1997. Another neural code? NeuroImage 5, 213-220.

Friston, K.J., 2000a. The labile brain: I. Neuronal transients and nonlinear coupling. Philos. Trans. R. Soc. Lond., B. Biol. Sci. 355, $215-236$.

Friston, K.J., 2000b. The labile brain: III. Transients and spatio-temporal receptive fields. Philos. Trans. R. Soc. Lond., B. Biol. Sci. 355, $253-265$ 
Friston, K., 2002. Functional integration and inference in the brain. Prog. Neurobiol. 68, 113-143.

Friston, K.J., Penny, W., Phillips, C., Kiebel, S., Hinton, G., Ashburner, J., 2002. Classical and Bayesian inference in neuroimaging: theory. NeuroImage 16, 465-483.

Friston, K.J., Harrison, L., Penny, W., 2003. Dynamic causal modelling. NeuroImage 19, 1273-1302.

Harrison, L.M., David, O., Friston, K.J., submitted for publication. Dynamic mean fields and ERP generation. Philos. Trans. R. Soc. Lond., B.

Haskell, E., Nykamp, D.Q., Tranchina, D., 2001. Population density methods for large-scale modelling of neuronal networks with realistic synaptic kinetics: cutting the dimension down to size. Network 12, 141-174.

Jansen, B.H., Rit, V.G., 1995. Electroencephalogram and visual evoked potential generation in a mathematical model of coupled cortical columns. Biol. Cybern. 73, 357-366.

Jansen, B.H., Agarwal, G., Hegde, A., Boutros, N.N., 2003. Phase synchronization of the ongoing EEG and auditory EP generation. Clin. Neurophysiol. 114, 79-85.

Jirsa, V.K., Kelso, J.A., 2000. Spatiotemporal pattern formation in neural systems with heterogeneous connection topologies. Phys. Rev. E: Stat., Phys., Plasmas, Fluids, Relat. Interdiscip. Topics 62, 8462-8465.

Kaneko, K., Tsuda, I., 2003. Chaotic itinerancy. Chaos 13, 926-936.

Klimesch, W., Schack, B., Schabus, M., Doppelmayr, M., Gruber, W., Sauseng, P., 2004. Phase-locked alpha and theta oscillations generate the P1-N1 complex and are related to memory performance. Brain Res. Cogn. Brain Res. 19, 302-316.

Kloeden, P.E., Platen, E., 1999. Numerical Solution of Stochastic Differential Equations. Springer-Verlag, Berlin.

Kolev, V., Yordanova, J., 1997. Analysis of phase-locking is informative for studying event-related EEG activity. Biol. Cybern. 76, 229-235.

Lachaux, J-P., Rodriguez, E., Martinerie, J., Varela, F.J., 1999. Measuring phase synchrony in brain signals. Hum. Brain Mapp. 8, 194-208.

Le Van Quyen, M., Foucher, J., Lachaux, J., Rodriguez, E., Lutz, A., Martinerie, J., Varela, F.J., 2001. Comparison of Hilbert transform and wavelet methods for the analysis of neuronal synchrony. J. Neurosci. Methods 111, 83-98.

Linsker, R., 1990. Perceptual neural organization: some approaches based on network models and information theory. Annu. Rev. Neurosci. 13, $257-281$.

Lopes da Silva, F.H., Hoeks, A., Smits, H., Zetterberg, L.H., 1974. Model of brain rhythmic activity. The alpha-rhythm of the thalamus. Kybernetik 15, 27-37.

Lopes da Silva, F.H., Pijn, J.P., Velis, D., Nijssen, P.C., 1997. Alpha rhythms: noise, dynamics and models. Int. J. Psychophysiol. 26, 237-249.

Lumer, E.D., Edelman, G.M., Tononi, G., 1997. Neural dynamics in a model of the thalamocortical system: I. Layers, loops and the emergence of fast synchronous rhythms. Cereb. Cortex 7, 207-227.

Makeig, S., Westerfield, M., Jung, T.P., Enghoff, S., Townsend, J., Courchesne, E., Sejnowski, T.J., 2002. Dynamic brain sources of visual evoked responses. Science 295, 690-694.

Miller, K.D., 2003. Understanding layer 4 of the cortical circuit: a model based on cat V1. Cereb. Cortex 13, 73-82.

Nunez, P.L., 1974. The brain wave equation: a model for the EEG. Math Biosci. 21, 279-297.
Penny, W.D., Kiebel, S.J., Kilner, J.M., Rugg, M.D., 2002. Event-related brain dynamics. Trends Neurosci. 25, 387-389.

Penny, W., Stephan, K., Mechelli, A., Friston, K., 2004. Comparing dynamic causal models. NeuroImage 22 (3), 1157-1172.

Pfurtscheller, G., Lopes da Silva, F.H., 1999. Event-related EEG/MEG synchronization and desynchronization: basic principles. Clin. Neurophysiol. 110, 1842-1857.

Rennie, C.J., Robinson, P.A., Wright, J.J., 2002. Unified neurophysical model of EEG spectra and evoked potentials. Biol. Cybern. 86, 457-471.

Robinson, P.A., Rennie, C.J., Wright, J.J., Bahramali, H., Gordon, E., Rowe, D.L., 2001. Prediction of electroencephalographic spectra from neurophysiology. Phys. Rev. E 63, 021903.

Robinson, P.A., Rennie, C.J., Rowe, D.L., 2002. Dynamics of large-scale brain activity in normal arousal states and epileptic seizures. Phys. Rev. E: Stat., Nonlin. Soft Matter Phys. 65, 041924.

Shah, A.S., Bressler, S.L., Knuth, K.H., Ding, M., Mehta, A.D., Ulbert, I., Schroeder, C.E., 2004. Neural dynamics and the fundamental mechanisms of event-related brain potentials. Cereb. Cortex 14, 476-483.

Stam, C.J., Pijn, J.P., Suffczynski, P., Lopes da Silva, F.H., 1999. Dynamics of the human alpha rhythm: evidence for non-linearity? Clin. Neurophysiol. 110, 1801-1813.

Suffczynski, P., Kalitzin, S., Pfurtscheller, G., Lopes da Silva, F.H., 2001. Computational model of thalamo-cortical networks: dynamical control of alpha rhythms in relation to focal attention. Int. J. Psychophysiol. 43 , $25-40$.

Tallon-Baudry, C., Bertrand, O., Delpuech, C., Pernier, J., 1996. Stimulus specificity of phase-locked and non-phase-locked $40 \mathrm{~Hz}$ visual responses in human. J. Neurosci. 16, 4240-4249.

Tass, P.A., 2003. Stochastic phase resetting of stimulus-locked responses of two coupled oscillators: transient response clustering, synchronization, and desynchronization. Chaos 13, 364-376.

Thomson, A.M., Deuchars, J., 1997. Synaptic interactions in neocortical local circuits: dual intracellular recordings in vitro. Cereb. Cortex 7, $510-522$.

Tsuda, I., 2001. Toward an interpretation of dynamic neural activity in terms of chaotic dynamical systems. Behav. Brain Sci. 24, 793-810.

Valdes, P.A., Jimenez, J.C., Riera, J., Biscay, R., Ozaki, T., 1999. Nonlinear EEG analysis based on a neural mass model. Biol. Cybern. $81,415-424$.

Van Rotterdam, A., Lopes da Silva, F.H., van den, E.J., Viergever, M.A., Hermans, A.J., 1982. A model of the spatial-temporal characteristics of the alpha rhythm. Bull Math Biol. 44, 283-305.

Varela, F., Lachaux, J-P., Rodriguez, E., Martinerie, J., 2001. The brainweb: phase synchronization and large-scale integration. Nat. Rev. Neurosci. 2, 229-239

Wendling, F., Bellanger, J.J., Bartolomei, F., Chauvel, P., 2000. Relevance of nonlinear lumped-parameter models in the analysis of depth-EEG epileptic signals. Biol. Cybern. 83, 367-378.

Wendling, F., Bartolomei, F., Bellanger, J.J., Chauvel, P., 2002. Epileptic fast activity can be explained by a model of impaired GABAergic dendritic inhibition. Eur. J. Neurosci. 15, 1499-1508.

Wilson, H.R., Cowan, J.D., 1972. Excitatory and inhibitory interactions in localized populations of model neurons. Biophys. J. 12, 1-24 